\title{
High Grade Intraepithelial Neoplasia
}

National Cancer Institute

\section{Source}

National Cancer Institute. High Grade Intraepithelial Neoplasia. NCI Thesaurus. Code C7348.

A precancerous neoplastic process that affects the squamous, glandular, or transitional cell epithelium without evidence of invasion. According to the degree of nuclear atypia, number of mitotic figures, and presence of architectural distortion, it is classified as grade II (moderate dysplasia) or grade III (severe dysplasia). 